

\section{Quantum beat between sunlight and single}

\section{photons}

Zhao-Chen Duan, ${ }^{\dagger, \downarrow}, \triangle$ Yu-Hao Deng, ${ }^{\dagger, \ddagger}, \triangle$ Ying Yu, ${ }^{*}$ Si Chen, ${ }^{\dagger, \ddagger}$ Jian Qin, ${ }^{\dagger, \ddagger}$ Hui Wang, ${ }^{\dagger, \ddagger}$ Xing Ding, ${ }^{\dagger, \ddagger}$ Li-Chao Peng, ${ }^{\dagger, \ddagger}$ Christian Schneider, ${ }^{\S}$ Da-Wei Wang, ${ }^{*, \|}$ Sven Höfling, ${ }^{\dagger, \S, \perp}$ Jonathan P. Dowling, ${ }^{\dagger, \#, @ ~ C h a o-Y a n g ~ L u, ~}{ }^{*, \dagger, \ddagger}$ and Jian-Wei $\operatorname{Pan}^{\dagger, \ddagger}$

$\dagger$ Shanghai Branch, Department of Modern Physics and National Laboratory for Physical Sciences at Microscale, University of Science and Technology of China, Shanghai 201315,

China

$\ddagger C A S$ Center for Excellence and Synergetic Innovation Center in Quantum Information and Quantum Physics, University of Science and Technology of China, Hefei, Anhui 230026, China

IState Key Laboratory of Optoelectronic Materials and Technologies, School of Electronics and Information Technology, School of Physics, Sun Yat-sen University, Guangzhou, Guangdong 510275, China

§Technische Physik, Physikalische Institut and Wilhelm Conrad Röntgen-Center for Complex Material Systems, Universität Würzburg, Am Hubland, D-97074 Würzburg,

Germany

||Department of Physics, Zhejiang University, Hangzhou, Zhejiang 310027, China

$\perp S U P A$, School of Physics and Astronomy, University of St. Andrews, St. Andrews KY16 9SS, United Kingdom

\#Hearne Institute for Theoretical Physics and Department of Physics and Astronomy, Louisiana State University, Baton Rouge, LA 70803, USA

@NYU-ECNU Institute for Physics at NYU Shanghai, Shanghai 200062, China $\triangle$ Contributed equally to this work

E-mail: yuying26@mail.sysu.edu.cn; dwwang@zju.edu.cn; cylu@ustc.edu.cn 


\begin{abstract}
We demonstrate fourth-order quantum beat between sunlight and single photons from a quantum dot. With a fast time-resolved detection system, we observed highvisibility quantum beat between the independent photons of different frequencies from the two astronomically separated light sources. The temporal dynamics of the beat oscillation indicates the coherent behavior of the interfering photons, and the raw visibility of two-photon interference shows violation of the classical limit with a frequency mismatch of three times the linewidth.
\end{abstract}

\title{
Keywords
}

quantum dots, quantum information processing, single photons, fourth-order interference, quantum beat 
When two identical photons are simultaneously incident on a balanced beam splitter (BS) from two input ports, they'll coalesce and leave the BS from the same output port. This counter-intuitive phenomenon of indistinguishable photons, known as Hong-Ou-Mandel (HOM) interference, manifests the bosonic nature of light. ${ }^{1}$ Besides its fundamental role in enriching our conceptual pool of experiments that demonstrate higher-order interference and coherence of light, and quantum foundations, ${ }^{2,3} \mathrm{HOM}$ interference has proven to have broad applications in quantum information processing (QIP). ${ }^{4-8}$ Driven by both fundamental interest and practical motivations, HOM interference has been demonstrated with various independent light sources, ${ }^{9-20}$ and very recently, sunlight with quantum dot (QD) singlephoton source. ${ }^{21}$

However, since quantum information technologies are booming quickly, there arise a practical need to use independent photon sources ${ }^{10,21-26}$ to build large-scale hybrid QIP networks through relays (like satellites) for real-life applications. ${ }^{27-29}$ Two major concerns arise from this extension. The first is the channel-induced decoherence and the second is the dissimilarity between photons at separate nodes of the network. Photon distinguishability in color (frequency) arises from both concerns through, e.g., Doppler effect from high-speed flying objects (like low-orbit satellites) and other inhomogeneous broadening in the emission spectra, and the frequency shifts they arise are significant ${ }^{30}$ (usually on the order of GHz for visible light and near infrared) compared to the linewidth of light fields. It is expected that quantum effects vanish only when there is noise or decoherence, which naturally suggests the hidden quantum interference in the case of frequency mismatching. It turns out that HOM interference manifests itself in the form of a quantum beat when the two photons are different in frequency, ${ }^{31,32}$ as long as the coincidence analysis is done in a space- or time-resolved way. This phenomenon bridges the gap between two-photon interference and frequency distinguishability between the photons by taking advantage of the time-energy uncertainty relationship of quantum mechanics, and its applications have been theoretically discussed ${ }^{33}$ and experimentally demonstrated with trapped atoms. ${ }^{30,34}$ To study the quantum beat, 
high demands must be met for the coherence properties of the photons and the time-resolved detection techniques. Furthermore, to distinguish the quantum from the classical beat effect, the photon source involved must be non-classical in its photon statistics. All these constraints limit previous studies on temporal quantum beat, within the scope of photons solely from laboratory single-photon sources, such as trapped atoms, ${ }^{35} \mathrm{QDs},{ }^{36}$ organic molecules, ${ }^{37}$ and nonlinear nanophotonics, ${ }^{38}$ which all have long coherence time. When it comes to the issue with classical light field, it is possible to observe a quantum interference. ${ }^{39}$ However, as in the present case of quantum beat with thermal light field (sunlight), additional attention must be paid to its photon statistics. The thermal light field with super-Poisson statistics is seen as a highly classical light field, and two photon interference experiment with thermal light field alone can never achieve a visibility higher than $1 / 3 .^{3}$

Here, we report on an experiment showing quantum beat between photons from the Sun $\left(1.5 \times 10^{8} \mathrm{~km}\right.$ away) and a QD single-photon source. The interest of the choice is beyond the issue of long-distance between independent sources, but it is also for probing the quantum nature of the thermal light field from distant astronomical objects. The luminance features of such objects are rarely looked into in a quantum mechanical way, and the lack of common history shared by the interfering photons forbids any possibility of pre-shared interaction or correlation between them. ${ }^{40}$ Our results, reported here, demonstrate for the first time quantum beat with a thermal light field, and opens new possibilities to study quantummechanical features of astronomical objects.

The experimental setup is shown in Figure 1, from the right part of which the BS has input modes $a$ and $b$, and output modes $c$ and $d$. We use Fock states $\left|n_{k}\right\rangle$ ( $n=0$ or 1 , and $k=a, b, c$, or $d$ ) to denote $n$ photons in the mode $k$, and we introduce photon creation and annihilation operators $\hat{a}_{k}^{\dagger}$ and $\hat{a}_{k}$ for the corresponding mode. Initially single photons from the two independent sources impinge simultaneously into the BS whose quantum state is represented as $\left|1_{a} 1_{b}\right\rangle$. Once detectors $c$ and $d$ are triggered respectively by one photon, the 
coincidence possibility gives

$$
\left\langle 1_{a} 1_{b}\left|\hat{a}_{c(d)}^{\dagger} \hat{a}_{d(c)}^{\dagger} \hat{a}_{d(c)} \hat{a}_{c(d)}\right| 1_{a} 1_{b}\right\rangle=\frac{1}{2}[1-\cos (\Delta \tau)]
$$

where $\Delta=\omega_{b}-\omega_{a}$ is the angular frequency detuning between the two photons and $\tau$ is the delay between the triggering times of two detectors, ${ }^{41}$ indicating a quantum beat with a period of $2 \pi / \Delta$ in the correlation measurement. The beat comes from the difference between the frequencies of the two photons, and when the detuning $\Delta=0$ it reduces to the conventional HOM interference. One remarkable feature of the interference is that the oscillation of the beat can reach $100 \%$ visibility, which is dramatically different from the case of intensity correlation interference with independent coherent light (or any classical light field), where the visibility is limited by no more than $50 \%$. ${ }^{42}$ We also note that the above analysis is just a simplified illustration of the basic principle. A detailed description and model of our experiment are developed in the following.

We collect photons from the Sun with an equatorial-mounted telescope. The photons are firstly coarsely filtered on the spectrum, and subsequently guided into the experimental platform through a single-mode optical fiber (SMF). The sunlight is coupled into SMF by a fiber collimator with an effective aperture of $\sim 4 \mathrm{~mm}$, and $\sim 1 \mathrm{~mW}$ of it is finally collected. The experiment is performed on clear days, with little cloud cover, and the optical window is monitored in real time to avoid fluctuations of the sunlight. To reveal the quantum nature of the highly classical thermal light from the Sun, it is crucial to interfere sunlight photons with photons from a non-classical light source. We deploy a state-of-the-art self-assembled InAs/GaAs QD embedded in a micro-pillar cavity as our single-photon source of near-unity indistinguishability and purity. ${ }^{43}$ The dot-cavity system is placed in a cryostat bath cooled down to $4.2 \mathrm{~K}$. The ultra-bright single photon source is resonantly excited by a Ti-sapphire picosecond laser with a repetition rate around $76 \mathrm{MHz}$, through a confocal microscope equipped with a cross-polarization filter, ${ }^{44}$ to collect the single-photon resonance fluorescence and eliminate 
the laser background. At $\pi$ pulse excitation single photons can be deterministically generated and a count rate of $\sim 14 \mathrm{MHz}$ is registered with the superconductor-nanowire single-photon detector (SNSPD). Our single photons have proven to be near-transform-limited, ${ }^{45}$ with a lifetime of $T_{1}=68.2(3) \mathrm{ps}$, a coherence time of $T_{2}=125(1) \mathrm{ps}$, and an indistinguishability plateau of 0.952(3) (Supporting Information).

The coherence between the two photons different in frequency relies on their mutual indistinguishability in almost all degrees of freedom, which are polarization, temporal, and spatial mode information. Firstly, since the sunlight is continuous in time, while QD single photons come in pulses synchronized with the laser period, they both differ drastically in the temporal degree of freedom. To meet this challenge, we use a fast SNSPD with a time resolution of $\sim 20 \mathrm{ps}$, to temporally resolve the interference pattern and suppress the contribution from the convolution noise of non-simultaneous events.

Secondly, we prepare the photons each in a single polarization state before interference by using a high-extinction-ratio polarizing beam splitter (PBS). At last, to achieve spatial mode indistinguishability, we project the single photons into the fundamental transverse Gaussian mode with SMFs.

Besides these distinguishability-erasing techniques in degrees of freedom other than frequency, the sunlight photons received at the ground are mixed in frequencies from a broad continuous solar spectrum that goes from ultraviolet to infrared light $(0.15-4 \mu \mathrm{m})$, while the single photons from the QD are narrowband in $\sim 893 \mathrm{~nm}$ with a full-width at half maximum (FWHM) of $\sim 2.5 \mathrm{GHz}$. For fine control in spectrum matching and detuning, we use an optical filtering system consisting of a band-pass filter mounted on equatorial telescope, a grating monochromator, and a series of etalons to filter the sunlight to monochromatic with FWHM of $\sim 1 \mathrm{GHz}$, while filtering the QD to a fixed narrower band with FWHM of $\sim 1 \mathrm{GHz}$, at the same time. The maximum photon flux for finely filtered sunlight here is estimated to be on the order of $10 \mathrm{MHz}$. The detuning between the two sources can thus be controlled by thermally tuning the temperature of the etalons in the sunlight path, providing in principle, 
flexible access to the full range of its spectrum (Supporting Information).

We now take into account of multi-photon events in the experiment. When two-photon (or higher photon number states) from either one of the sources enters the BS, they behave independently and trigger coincidence events without any interference feature, decreasing the observed visibility. It is remarkable that for sunlight the issue manifests itself in two ways. Firstly, multi-photon events from the Sun come continuously at all times, even when there are no single photons from the QD, leading to abundant coincidence events. We discard these joint counts by electrically gating our detector ON only at the time when single photons come from the QD, whose emission is synchronized with the laser. In the experiment, the gating window is opened for $1 \mathrm{~ns}$ each excitation period and can suppress the contribution of the multi-photon events from the Sun by a factor of $\sim 13$. It is worth noting that both sunlight and single-photon resonance fluorescence finally go through $1 \mathrm{GHz}$ etalons so that their coherence time have been lengthened, we choose 1 ns gating time instead of shorter time to match the coherence time of filtered photons. The gating results in a modulation of the counting in each detector with a square wave signal.

Secondly and more importantly, the probability of $n$-photon events that satisfies a thermal distribution. While the multi-photon events can be decreased by reducing the average photon number (light intensity) $\bar{n}$, so that the theoretical two-photon interference visibility with an ideal single-photon source can achieve $100 \%$ in principle, ${ }^{46}$ it is not practical to have a $100 \%$ pure single-photon source. As a result, there is a subtle trade-off between a too strong and a too weak thermal light field. To address this challenge, we have developed a theoretical model to look into the problem and extract a neat formula for the raw visibility (Supporting Information)

$$
\frac{V_{\text {corr }}}{V_{\text {raw }}}=1+\frac{T_{G}}{2 T_{P}} \frac{I_{\mathrm{Sun}}}{I_{\mathrm{QD}}} g_{\mathrm{Sun}}^{(2)}(0)+\frac{T_{P} \sigma_{\mathrm{QD}}}{2} \frac{I_{\mathrm{QD}}}{I_{\mathrm{Sun}}} g_{\mathrm{QD}}^{(2)}(0),
$$

where $V_{\text {raw }}$ is the raw visibility, $V_{\text {corr }}$ is the visibility corrected from multi-photon events, $T_{P}$ is the laser pulse period, $T_{G}$ is the width of the time-gating window, $g_{\mathrm{Sun}(\mathrm{QD})}^{(2)}(0)$ is the normalized second-order correlation function of sunlight (single photons) at zero-delay, $\sigma_{\mathrm{QD}}$ 


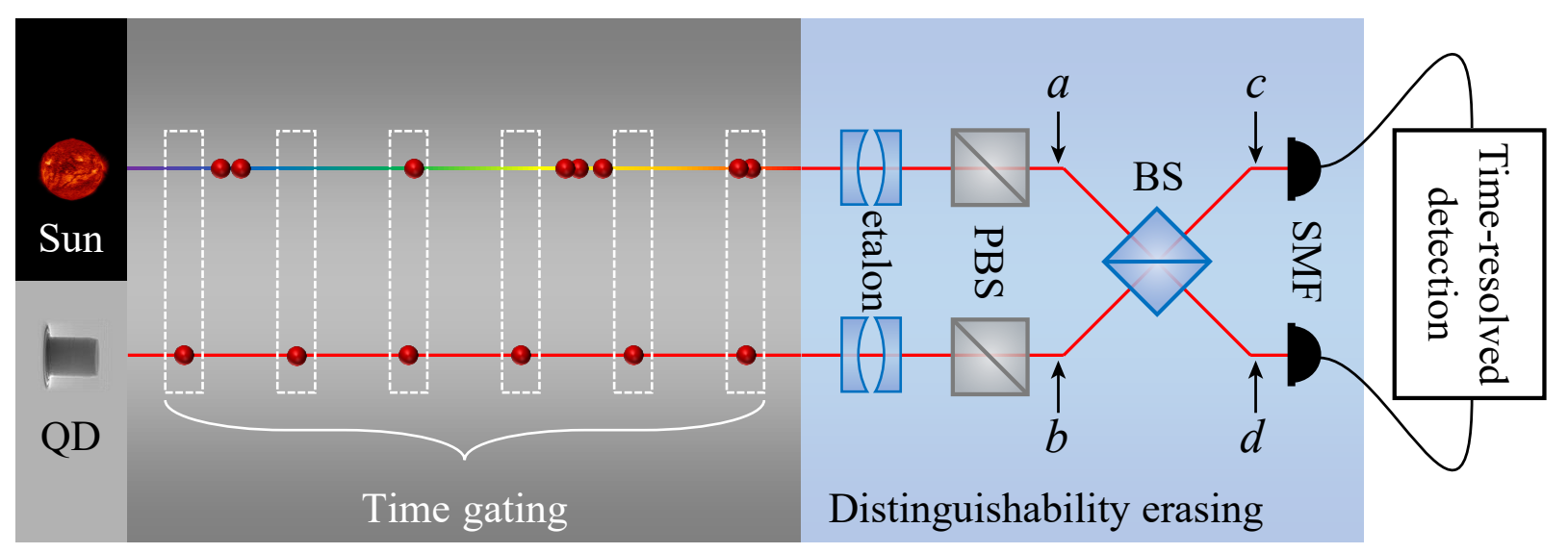

Figure 1: Experimental setup. SMFs project the photons from both sources into identical Gaussian fundamental spatial modes. The photons then enter an independent spectral filtering series composed of thermally controlled etalons and gratings. Finally they are polarized with a PBS and their mutual distinguishability can be determined by the orientation of the half-wave plate (HWP). The BS has input modes $a$ and $b$, and output modes $c$ and $d$, and initially photons from two independent sources are present in the quantum state of $\left|1_{a} 1_{b}\right\rangle$. The detectors are gated by a square wave signal synchronized with the excitation laser pulse to suppress the contribution of the multi-photon events from the Sun. BS, balanced beam splitter; PBS, polarizing beam splitter; SMF, single-mode optical fiber.

is the linewidth of the QD single photons, and $I_{\mathrm{Sun}(\mathrm{QD})}$ is the intensity of sunlight (single photons). As seen from Equation (2), the raw visibility is sensitively dependent on the purity of the QD single-photon source. Guided by the model, the intensities of sunlight and single photons are set to be $\sim 200 \mathrm{kHz}$ and $\sim 150 \mathrm{kHz}$ to achieve high visibility.

We now introduce the results of the quantum beat thus obtained. We first look into the zero-detuning situation, because in this case the conventional HOM interference is expected. In Figure 2a we show the measured coincidence at $\Delta=0$ for both the indistinguishable situation (parallel polarization) and distinguishable situation (cross polarization), and the classical limit 0.5 is marked with the dashed grey line. We note that the results we obtained are raw data without any subtraction of the background. The results display a remarkable HOM dip at zero-time delay with a raw visibility of $0.73(3)$, significantly above the classical limit, unambiguously revealing the quantum nature of the interference. We attribute the non-ideal visibility at zero-time delay to the contribution of multi-photon events from both 

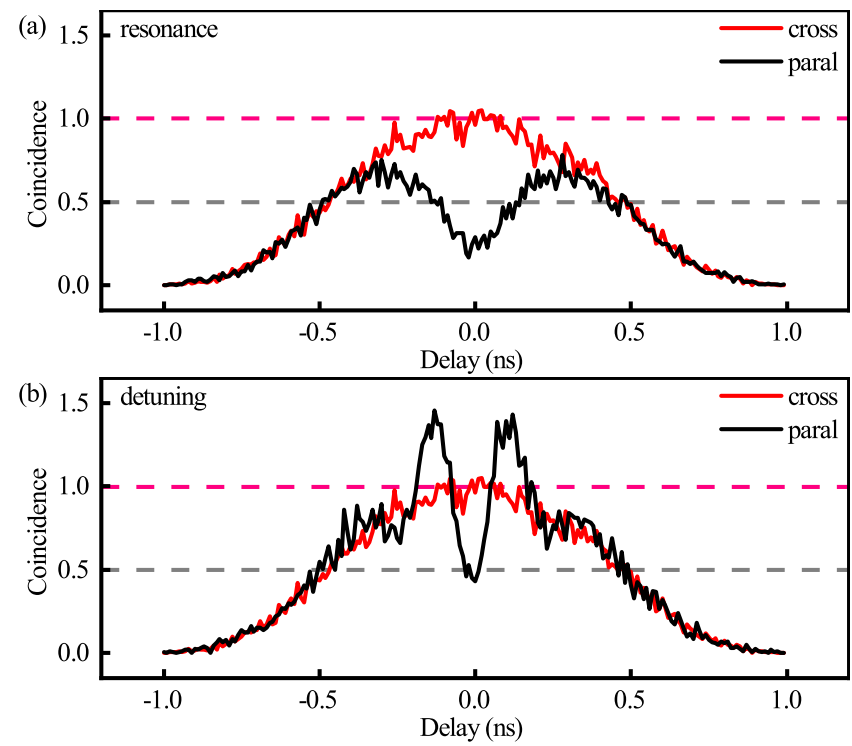

Figure 2: Raw experimental data of two-photon correlation measurement between sunlight and single photons at (a) zero-detuning, and (b) $4 \mathrm{GHz}$ detuning, with time gating of $1 \mathrm{~ns}$. Correlation functions are analyzed on the time-tagged data registered by a TDC with a timebin of $10 \mathrm{ps}$, and normalized according to accumulation time. The intensities of sunlight and single photons are set to be $\sim 200 \mathrm{kHz}$ and $\sim 150 \mathrm{kHz}$. The correlation count rate for the 10 ps time-bin around zero-delay is $\sim 0.14 \mathrm{~Hz}$ (cross-polarization), and the overall correlation count rate displayed in the figure, is $\sim 13.3 \mathrm{~Hz}$. A pronounced minimum shown at zero-delay for parallel polarization, and no interfering feature for cross polarization. In the detuning regime of $4 \mathrm{GHz}$, the quantum beat effect is clearly displayed. The visibility is calculated by $V_{\text {raw }}=1-g_{\|}^{(2)}(0) / g_{\perp}^{(2)}(0)$, where $g_{\|(\perp)}^{(2)}(0)$ is normalized correlation function for parallel (cross) polarization at zero-delay.

sources, convolution noise from non-simultaneous coincidences, non-ideal indistinguishability in polarization, and the minor unbalance of the BS. The measured quantum beat effect in coincidence for $\Delta=2 \pi \times 4 \mathrm{GHz}$ is displayed in Figure $2 \mathrm{~b}$. The correlation displays a pronounced oscillation from the minimum at zero-time delay, going up to the first maximum at $|\tau| \approx 130$ ps. We extract a raw visibility of the quantum beat oscillation of $0.56(4)$, well above the classical limit. The interference at a detuning of three times the FWHM of both sources still displays a clear beat fringe. The non-unity visibility of the quantum beat is mainly due to the multi-photon events and time convolution noise from the detectors, since the oscillation period of $\sim 250 \mathrm{ps}$ is only about one order larger than the time-resolution of our fast SNSPD. 
We have demonstrated the quantum nature of two-photon interference and beat oscillations between photons from the Sun and a QD. In the following, we examine the effect in a more detailed way. The contribution of the registered coincidence can be decomposed to three major parts that add up linearly under the low-light intensity regime, where the possibility of events involving more than two photons is $\sim 1.8 \times 10^{-8}$, which is neglectable compared to single-photon possibility with a relative ratio of the order of $10^{-5}$,

$$
\mathcal{G}^{(2)}(\tau)=\mathcal{G}_{\mathrm{Sun}}^{(2)}(\tau)+\mathcal{G}_{\text {int }}^{(2)}(\tau)+\mathcal{G}_{\mathrm{QD}}^{(2)}(\tau)
$$

where $\mathcal{G}^{(2)}(\tau)$ indicates the joint count rates with a triggering time difference $\tau$ between detectors $c$ and $d$. The second term on the right-hand side indicates the pure interference correlation between one photon from the Sun and one photon from the QD, while the two other terms are the self-correlation of both sources caused by multi-photon events background that decreases the two-photon quantum interference of interest, which can be corrected from the raw counts.

We characterize the photon statistics properties of both sources by measuring their second-order self-correlation functions $\mathcal{G}_{\text {Sun }}^{(2)}(\tau)$ and $\mathcal{G}_{\mathrm{QD}}^{(2)}(\tau)$ with a Hanbury Brown-Twiss (HBT) setup, ${ }^{47}$ For measuring second-order self-correlation of the QD, we close the input path from the Sun so that photons impinge on the BS only from the QD. The second-order self-correlation of the QD single photon source is shown in Figure 3a, which displays typical anti-bunching at $\tau=0$, indicating a good single-photon purity of $g_{\mathrm{QD}}^{(2)}(0)=0.013(1)$. For the self-correlation measurement of the sunlight, we alternate the path-switching setting. In Figure 3b, we show the second-order self-correlation function of sunlight, which displays typical bunching at $\tau=0$, a characteristic of thermal light. Note that the triangular form of the modulation of the continuous coincidence of the sunlight background is attributed to the time gating. The $g_{\text {Sun }}^{(2)}(0)$ is calculated to be $1.96(8)$, which is much close the ideal value of 2. This ideal value benefits mainly from our more advanced fast time-resolved detection 


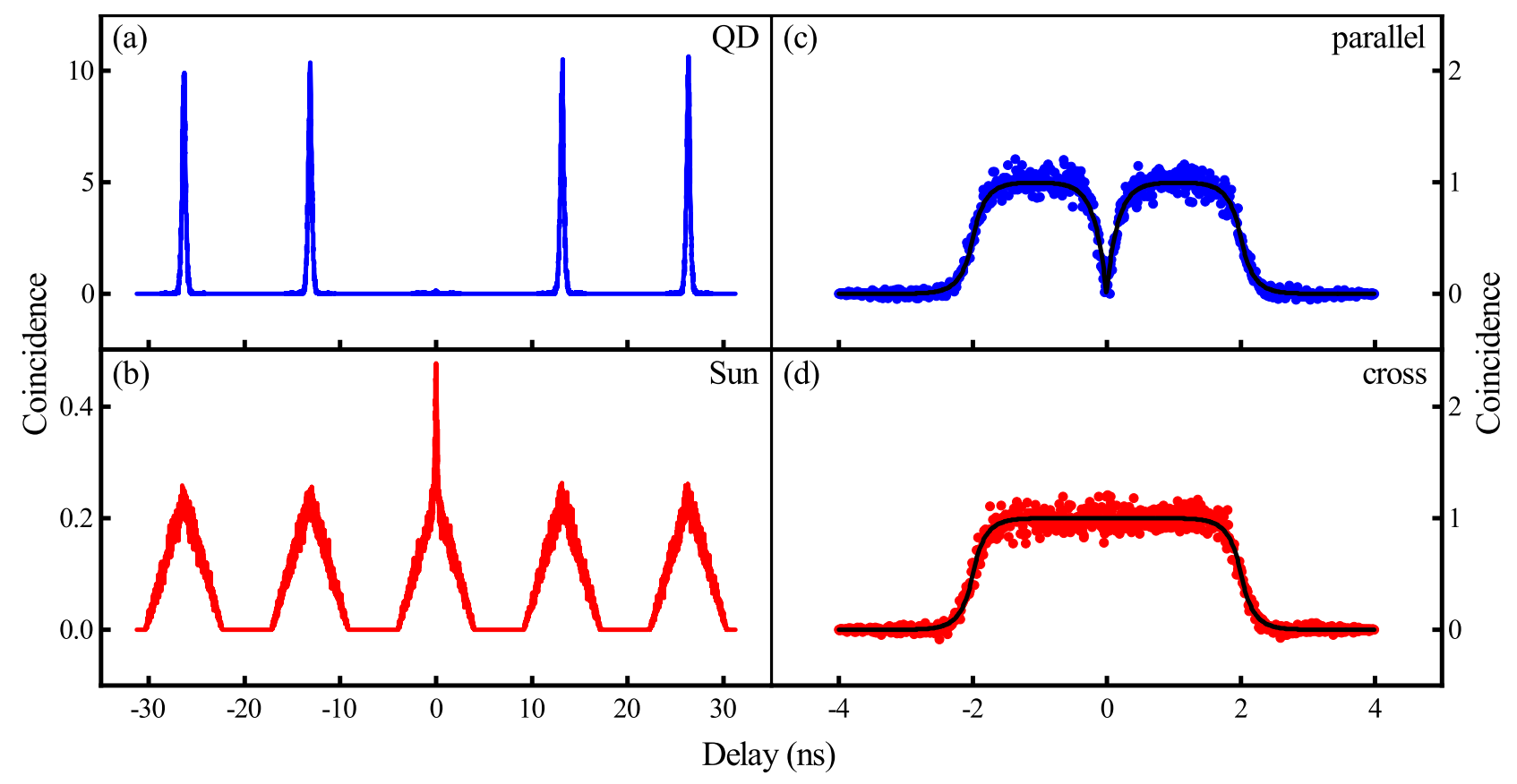

Figure 3: Second-order correlation functions with the time gating of $4 \mathrm{~ns}$. (a,b) Selfcorrelations of (a) single-photon resonance fluorescence $\mathcal{G}_{\mathrm{QD}}^{(2)}(\tau)$ and (b) sunlight $\mathcal{G}_{\text {Sun }}^{(2)}(\tau)$. $(\mathrm{c}, \mathrm{d})$ The pure interference correlations between one photon from the Sun and the other from a QD $\mathcal{G}_{\text {int }}^{(2)}(\tau)$, with two interfering photons in (c) parallel polarization $\mathcal{G}_{\|}^{(2)}(\tau)$ and (d) cross polarization $\mathcal{G}_{\perp}^{(2)}(\tau)$. It shows a plateau on the $\mathcal{G}_{\perp}^{(2)}(\tau)$ data around zero-delay at the condition of $T_{G} \gg 1 / \sigma$, and all correlation data are divided by data accumulation time. The normalized $g_{\mathrm{QD}}^{(2)}(0)$ and $g_{\mathrm{Sun}}^{(2)}(0)$ are calculated to be $0.013(1)$ and $1.96(8)$, and $\sigma$ is extracted by fitting $\mathcal{G}_{\|}^{(2)}(\tau)$ data.

system and fine filtering series. Divided by data accumulation time, the second-order selfcorrelation functions of both sources correspond to the multi-photon events measured in the quantum beat recording when both paths are opened. By using data corrected from these backgrounds, the pure interference correlation $\mathcal{G}_{\text {int }}^{(2)}(\tau)$, as shown in Figure $3 \mathrm{c}$ and 3d, with two photons parallel $\mathcal{G}_{\|}^{(2)}(\tau)$ and cross $\mathcal{G}_{\perp}^{(2)}(\tau)$ in polarization are revealed.

We show the quantum beat for different detuning from $\Delta=2$ to $8 \mathrm{GHz}$ in Figure 4 . The dots are the data points, while the solid curves are fittings based on the modelling of the quantum beat effect (Supporting Information)

$$
\mathcal{G}_{\|}^{(2)}(\tau)=\mathcal{G}_{\perp}^{(2)}(\tau)\left[1-V \mathrm{e}^{-\sigma|\tau|} \cos (\Delta \tau)\right]
$$




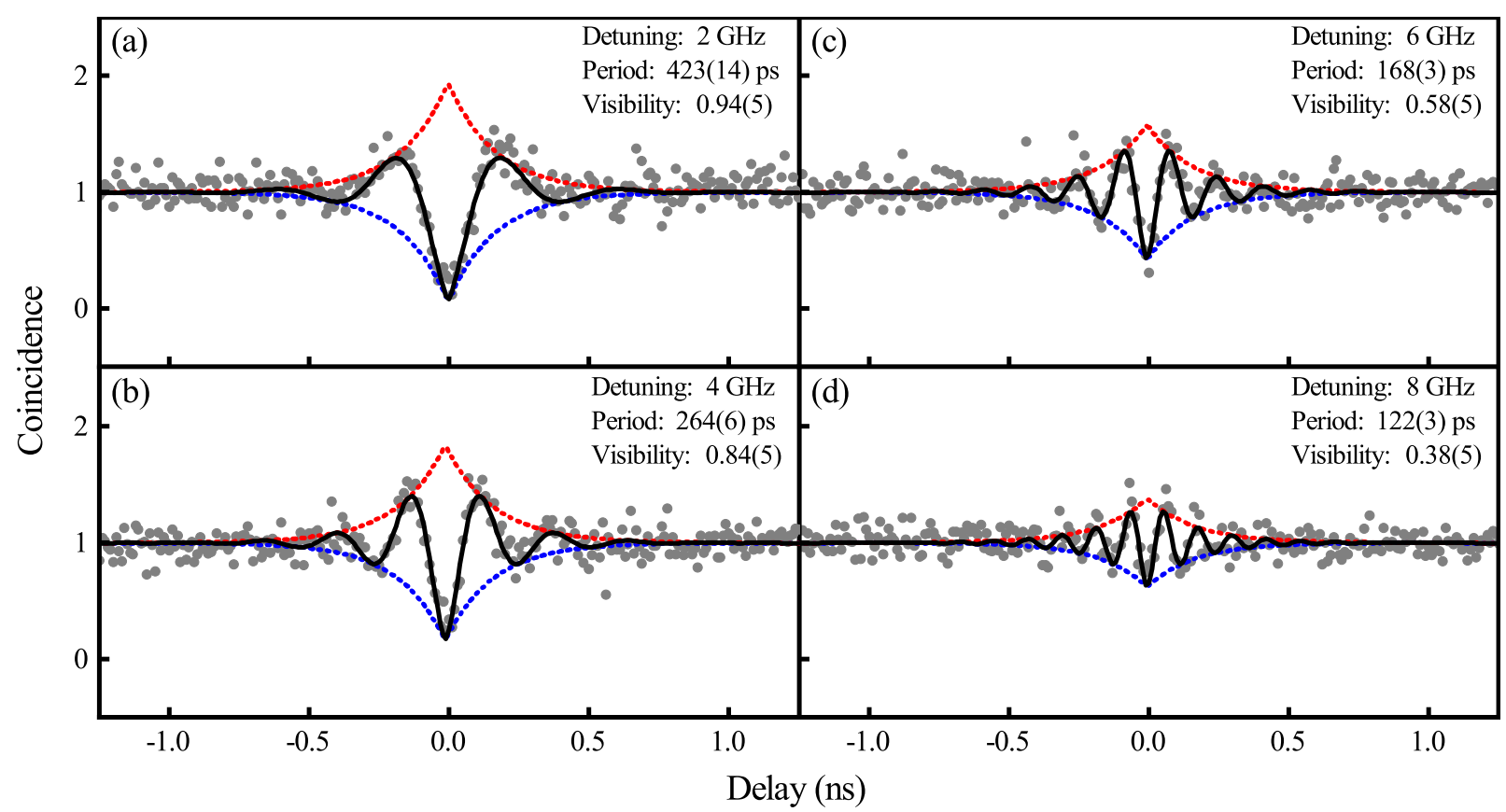

Figure 4: (a-d) Normalized two-photon coincidence counts with the detuning of two photons increases from 2 to $8 \mathrm{GHz}$. Two interfering photons are parallel in polarization. The data are fitted according to the square bracket term of Equation (4) with fixed $\sigma$ extracted from Figure 3c. A pronounced oscillation appears between envelopes (dashed curves). The calculated period, marked on the top-right corner of each plot, shows small deviations from the expected, and the visibility decreases from unity as the period gets closer to the time jitter of the detectors.

At the condition that the gating time, used in Figure 3 and 4 , is chosen to be $T_{G}=4 \mathrm{~ns}$ to satisfy $T_{G} \gg 1 / \sigma$, a plateau is observed on the $\mathcal{G}_{\perp}^{(2)}(\tau)$ data shown in Figure 3d, and $\sigma$ can be easily extracted to be $5.89(17) \mathrm{GHz}$ by fitting the $\mathcal{G}_{\|}^{(2)}(\tau)$ data shown in Figure 3c. The visiblility $V$ in Equation (4) corresponds to corrected visibility. For all detuning, the joint coincidence displays an oscillation starting at a minimum from $\tau=0$ to the first peak at $\tau \approx \pi / \Delta$. The amplitude of oscillation damps according to the coherence time $1 / \sigma$ of the photons from both sources, and the envelope of the oscillation is marked on the plots with dashed curves, which fits the data well, indicating pronounced coherence between the frequency-mismatched photons. We emphasize that as the detuning increases, the oscillation period shortened (from $\sim 500 \mathrm{ps}$ to $\sim 125 \mathrm{ps}$ ) so the visibility reduces from the convolution noise, which is due to the limited time-resolution of the detectors ( $\sim 20 \mathrm{ps})$. The coherence 
time of single photons, calculated from our previous literature, ${ }^{21}$ is $\sim 340 \mathrm{ps}$, which is longer than that extracted from Fourier transform of QD spectrum due to the spectral filtering. It is expected that the temporal dynamics can be better time-resolved with single photons of longer coherence time. One possible way to increase the coherence time is to use narrower etalon to filter the single photons as a trade-off with high photon flux, or the other way by using narrow line-width single photon sources such as trapped atoms.

In conclusion, we demonstrated the fourth-order quantum beat between thermal light field from the Sun, and single photons from the solid-state QD. With a frequency mismatch between the independent photons, the quantum beat of the two-photon interference is observed in the coincidence measurement at the output ports of the BS. The oscillation of the quantum beat has a period corresponding to the frequency difference between the interfering photons. The interference is significant only for photons registered in the coherence-time window. At zero-detuning, the observation reduces to the famous HOM interference, where two photons bunch at the BS, indicating remarkable coherence and indistinguishability between the photons from dissimilar sources. The observed non-unity visibility of the interference fringes is mainly ascribed to multi-photon events and limited time-resolution of our detectors. We expect that by implementing a single-photon source with better purity, a nearly perfect two-photon interference can be obtained. The time resolution of the quantum beat can be improved by using single photons with longer temporal durations, using narrower filtering, or choosing light sources with a longer lifetime. With finer temporal probes into the quantum beat effect, the experiment can be used to investigate the quantum characteristics of the starlight. ${ }^{48,49}$ One can conceive probing the second-order correlation properties of photons in other wavelengths of the Sun based on the reported techniques, in particular the special lines in the spectrum. We conjecture their coherence properties could be very different in cases like when there are dramatic changes in the solar environment. Recent efforts in applying quantum beat for QIP between dissimilar photon sources, ${ }^{33}$ from establishment and characterization of multiphoton networks, ${ }^{30,48,50}$ to demonstrations of quantum 
computational speed up, ${ }^{34,51}$ especially in cases at a large scale, can also benefit from our approach.

\section{Acknowledgement}

This work is supported by the National Natural Science Foundation of China, the Chinese Academy of Sciences, the Anhui Initiative in Quantum Information Technologies, the Science and Technology Commission of Shanghai Municipality, the National Fundamental Research Program, the State of Bavaria, and the US National Science Foundation.

\section{Supporting Information Available}

The Supporting Information is available free of charge on the ACS Publications website.

- Filename: beat-si-001.pdf

\section{References}

(1) Hong, C. K.; Ou, Z. Y.; Mandel, L. Phys. Rev. Lett. 1987, 59, 2044-2046.

(2) Pan, J.-W.; Chen, Z.-B.; Lu, C.-Y.; Weinfurter, H.; Zeilinger, A.; Żukowski, M. Rev. Mod. Phys. 2012, 84, 777-838.

(3) Paul, H. Rev. Mod. Phys. 1986, 58, 209-231.

(4) Ou, Z. Y.; Mandel, L. Phys. Rev. Lett. 1988, 61, 50-53.

(5) Shih, Y. H.; Alley, C. O. Phys. Rev. Lett. 1988, 61, 2921-2924.

(6) Bennett, C. H.; Brassard, G.; Crépeau, C.; Jozsa, R.; Peres, A.; Wootters, W. K. Phys. Rev. Lett. 1993, 70, 1895-1899. 
(7) Briegel, H.-J.; Dür, W.; Cirac, J. I.; Zoller, P. Phys. Rev. Lett. 1998, 81, 5932-5935.

(8) Lo, H.-K.; Curty, M.; Qi, B. Phys. Rev. Lett. 2012, 108, 130503.

(9) de Riedmatten, H.; Marcikic, I.; Tittel, W.; Zbinden, H.; Gisin, N. Phys. Rev. A 2003, 67, 022301.

(10) Kaltenbaek, R.; Blauensteiner, B.; Żukowski, M.; Aspelmeyer, M.; Zeilinger, A. Phys. Rev. Lett. 2006, 96, 240502.

(11) Beugnon, J.; Jones, M. P.; Dingjan, J.; Darquié, B.; Messin, G.; Browaeys, A.; Grangier, P. Nature 2006, 440, 779-782.

(12) Chanelière, T.; Matsukevich, D. N.; Jenkins, S. D.; Lan, S.-Y.; Zhao, R.; Kennedy, T. A. B.; Kuzmich, A. Phys. Rev. Lett. 2007, 98, 113602.

(13) Maunz, P.; Moehring, D. L.; Olmschenk, S.; Younge, K. C.; Matsukevich, D. N.; Monroe, C. Nat. Phys. 2007, 3, 538-541.

(14) Lang, C.; Eichler, C.; Steffen, L.; Fink, J. M.; Woolley, M. J.; Blais, A.; Wallraff, A. Nat. Phys. 2013, 9, 345-348.

(15) Bernien, H.; Childress, L.; Robledo, L.; Markham, M.; Twitchen, D.; Hanson, R. Phys. Rev. Lett. 2012, 108, 043604.

(16) Sipahigil, A.; Goldman, M. L.; Togan, E.; Chu, Y.; Markham, M.; Twitchen, D. J.; Zibrov, A. S.; Kubanek, A.; Lukin, M. D. Phys. Rev. Lett. 2012, 108, 143601.

(17) Sipahigil, A.; Jahnke, K. D.; Rogers, L. J.; Teraji, T.; Isoya, J.; Zibrov, A. S.; Jelezko, F.; Lukin, M. D. Phys. Rev. Lett. 2014, 113, 113602.

(18) Santori, C.; Fattal, D.; Vučković, J.; Solomon, G. S.; Yamamoto, Y. Nature 2002, 419, $594-597$. 
(19) Flagg, E. B.; Muller, A.; Polyakov, S. V.; Ling, A.; Migdall, A.; Solomon, G. S. Phys. Rev. Lett. 2010, 104, 137401.

(20) Patel, R. B.; Bennett, A. J.; Farrer, I.; Nicoll, C. A.; Ritchie, D. A.; Shields, A. J. Nat. Photonics 2010, 4, 632-635.

(21) Deng, Y.-H.; Wang, H.; Ding, X.; Duan, Z.-C.; Qin, J.; Chen, M.-C.; He, Y.; He, Y.-M.; Li, J.-P.; Li, Y.-H., et al. Phys. Rev. Lett. 2019, 123, 080401.

(22) Rarity, J. G.; Tapster, P. R.; Loudon, R. J. Opt. B: Quantum Semiclassical Opt. 2005, 7, S171-S175.

(23) Wiegner, R.; von Zanthier, J.; Agarwal, G. S. J. Phys. B: At., Mol. Opt. Phys. 2011, 44,055501 .

(24) Li, X.; Yang, L.; Cui, L.; Ou, Z. Y.; Yu, D. Opt. Express 2008, 16, 12505-12510.

(25) Bennett, A. J.; Patel, R. B.; Nicoll, C. A.; Ritchie, D. A.; Shields, A. J. Nat. Phys. 2009, 5, 715-717.

(26) Polyakov, S. V.; Muller, A.; Flagg, E. B.; Ling, A.; Borjemscaia, N.; van Keuren, E.; Migdall, A.; Solomon, G. S. Phys. Rev. Lett. 2011, 107, 157402.

(27) Liao, S.-K.; Cai, W.-Q.; Handsteiner, J.; Liu, B.; Yin, J.; Zhang, L.; Rauch, D.; Fink, M.; Ren, J.-G.; Liu, W.-Y., et al. Phys. Rev. Lett. 2018, 120, 030501.

(28) Simon, C. Nat. Photonics 2017, 11, 678-680.

(29) Agnesi, C.; Vedovato, F.; Schiavon, M.; Dequal, D.; Calderaro, L.; Tomasin, M.; Marangon, D. G.; Stanco, A.; Luceri, V.; Bianco, G., et al. Philos. Trans. R. Soc., A 2018, 376, 20170461.

(30) Zhao, T.-M.; Zhang, H.; Yang, J.; Sang, Z.-R.; Jiang, X.; Bao, X.-H.; Pan, J.-W. Phys. Rev. Lett. 2014, 112, 103602. 
(31) Ou, Z. Y.; Mandel, L. Phys. Rev. Lett. 1988, 61, 54-57.

(32) Legero, T.; Wilk, T.; Kuhn, A.; Rempe, G. Appl. Phys. B: Lasers Opt. 2003, 7r, $797-802$.

(33) Metz, J.; Barrett, S. D. Phys. Rev. A 2008, 77, 042323.

(34) Wang, X.-J.; Jing, B.; Sun, P.-F.; Yang, C.-W.; Yu, Y.; Tamma, V.; Bao, X.-H.; Pan, J.-W. Phys. Rev. Lett. 2018, 121, 080501.

(35) Legero, T.; Wilk, T.; Hennrich, M.; Rempe, G.; Kuhn, A. Phys. Rev. Lett. 2004, 93, 070503.

(36) Weber, J. H.; Kambs, B.; Kettler, J.; Kern, S.; Maisch, J.; Vural, H.; Jetter, M.; Portalupi, S. L.; Becher, C.; Michler, P. Nat. Nanotechnol. 2018, 14, 23-26.

(37) Lettow, R.; Rezus, Y.; Renn, A.; Zumofen, G.; Ikonen, E.; Götzinger, S.; Sandoghdar, V. Phys. Rev. Lett. 2010, 104, 123605.

(38) Li, Q.; Singh, A.; Lu, X.; Lawall, J.; Verma, V.; Mirin, R.; Nam, S. W.; Srinivasan, K. Phys. Rev. Appl. 2019, 12, 054054.

(39) Hong, C. K.; Ou, Z. Y.; Mandel, L. Phys. Rev. A 1988, 37, 3006-3009.

(40) Pittman, T. B.; Franson, J. D. Phys. Rev. Lett. 2003, 90, 240401.

(41) Yang, L.-K.; Cai, H.; Peng, T.; Wang, D.-W. J. Phys. B: At., Mol. Opt. Phys. 2018, $51,125501$.

(42) Scully, M. O.; Zubairy, M. S. Quantum optics; Cambridge University Press: Cambridge, 1997.

(43) Ding, X.; He, Y.; Duan, Z.-C.; Gregersen, N.; Chen, M.-C.; Unsleber, S.; Maier, S.; Schneider, C.; Kamp, M.; Höfling, S., et al. Phys. Rev. Lett. 2016, 116, 020401. 
(44) He, Y.-M.; He, Y.; Wei, Y.-J.; Wu, D.; Atatüre, M.; Schneider, C.; Höfling, S.; Kamp, M.; Lu, C.-Y.; Pan, J.-W. Nat. Nanotechnol. 2013, 8, 213-217.

(45) Wang, H.; Duan, Z.-C.; Li, Y.-H.; Chen, S.; Li, J.-P.; He, Y.-M.; Chen, M.-C.; He, Y.; Ding, X.; Peng, C.-Z., et al. Phys. Rev. Lett. 2016, 116, 213601.

(46) Ou, Z. Y. Phys. Rev. A 1988, 37, 1607-1619.

(47) Hanbury Brown, R.; Twiss, R. Q. Nature 1956, 178, 1046-1048.

(48) Legero, T.; Wilk, T.; Kuhn, A.; Rempe, G. Adv. At., Mol., Opt. Phys. 2006, 53, 253-289.

(49) Wasilewski, W.; Kolenderski, P.; Frankowski, R. Phys. Rev. Lett. 2007, 99, 123601.

(50) Laibacher, S.; Tamma, V. Phys. Rev. A 2018, 98, 053829.

(51) Laibacher, S.; Tamma, V. Phys. Rev. Lett. 2015, 115, 243605. 


\section{Graphical TOC Entry}

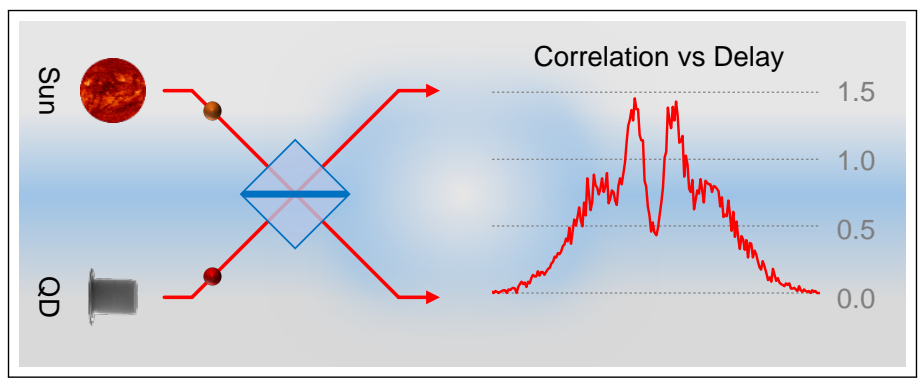

